\title{
Fetal body stalk malformation-a rare case report
}

\section{Roshini Balachandran*, Sunil Kumar Samal}

\begin{abstract}
Department of Obstetrics and Gynaecology, Mahatma Gandhi Medical College and Research Institute, Puducherry,
\end{abstract} Tamil Nadu, India

Received: 28 October 2020

Accepted: 05 December 2020

\section{*Correspondence:}

Dr. Roshini Balachandran,

E-mail: bsroshini@gmail.com

Copyright: ( ) the author(s), publisher and licensee Medip Academy. This is an open-access article distributed under the terms of the Creative Commons Attribution Non-Commercial License, which permits unrestricted non-commercial use, distribution, and reproduction in any medium, provided the original work is properly cited.

\begin{abstract}
Body stalk anomaly is a rare sporadic defect of the abdominal wall with evisceration of the thoracic and abdominal organs. Body stalk anomaly is reported in about 1 in 7500 births. Here we report about a primigravida with a fetus with posterior encephalocele, hyperextended cervical spine, kyphoscoliosis, large ventral body wall defect involving the entire thorax and abdomen with herniation of vital organs-heart, liver and intestine into the amniotic cavity. Prognosis of the fetal outcome was explained and medical termination of pregnancy was carried out. Body stalk malformation is a lethal condition either in utero or in early neonatal period which can be diagnosed in early trimester ultrasound. The association of chromosomal abnormalities and genetic syndromes in this condition is rare and the risk of recurrence in next pregnancy is less.
\end{abstract}

Keywords: Encephalocele, Abdominal wall, Omphalocele

\section{INTRODUCTION}

Body stalk anomaly is a rare sporadic defect of the abdominal wall with evisceration of the thoracic and abdominal organs. Body stalk anomaly is reported in about 1 in 7500 births in 10-14 weeks of gestation. ${ }^{1}$ The pathogenesis of body stalk defects may be due to abnormal closure of the body wall and abnormal embryonic folding during the transformation of the trilaminar embryo into a cylindrical embryo in first 4 weeks of embryogenesis. ${ }^{1}$ Other causes include early amnion rupture with amniotic band syndrome and reduction of the blood flow in early gestation. It is characterised by the absent or short umbilical cord with extruded abdominal organs outside of the abdominal cavity, kyphoscoliosis, limb deformities and craniofacial defects. Foetus affected with body stalk deformity usually have normal karyotype and with increased serum alpha feto-protein levels. ${ }^{2}$ This defect is usually diagnosed in the first trimester with the help of ultrasound. The differential diagnosis may be other abdominal wall defects in a fetus namely amniotic band syndrome, pantology of Cantrell, gastroschisis, omphalocele, exstrophy of bladder or OEIS complex.

\section{CASE REPORT}

26 years old Primigravida with a non-consanguineous marriage, for 2 years, conceived spontaneously had an uneventful first trimester. On anomaly scan at 18 weeks of gestation it was observed that, the foetus had multiple congenital malformations including occipital encephalocele, hyperextended cervical spine, kyphoscoliosis, large ventral body wall defect involving the entire thorax and upper abdomen with herniation of vital organs-heart, liver and intestine into the amniotic cavity (Figure 1, 2). Cord was short and seen inserted below the defect. A provisional diagnosis of large ventral wall defect with occipital encephalocele suggestive of body stalk malformation was made. The couple was counselled regarding the prognosis of this condition and they wanted termination of pregnancy. As the association of chromosomal anomalies and genetic syndromes is not increased with this condition, prenatal genetic tests were not offered. After MTP, a female foetus weighing $60 \mathrm{gm}$ 
was expelled. Post expulsion findings were large occipital encephalocele, large ventral wall defect involving thorax and upper abdomen with extrusion of heart, liver and small bowel. Cord was short and seen attached below the defect. The left big toe was absent (Figure 3). Parents refused fetal autopsy because of religious reasons.

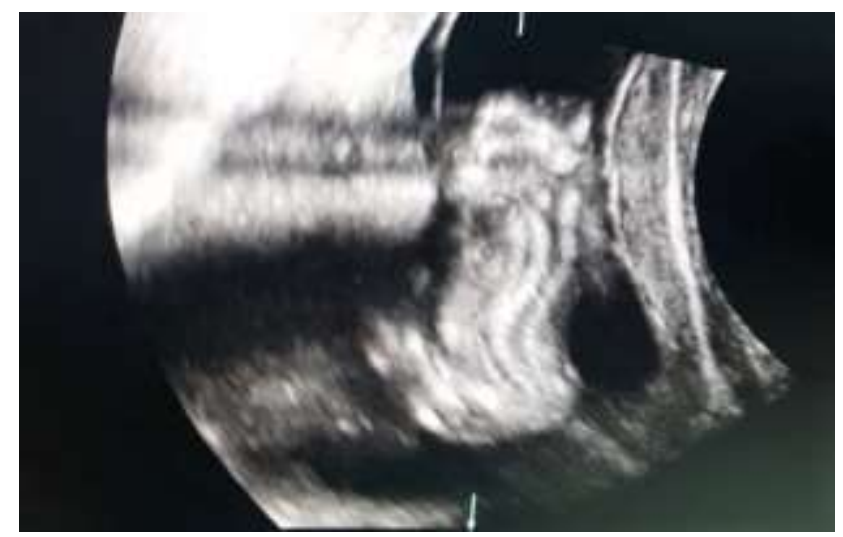

Figure 1: Kyphoscoliosis

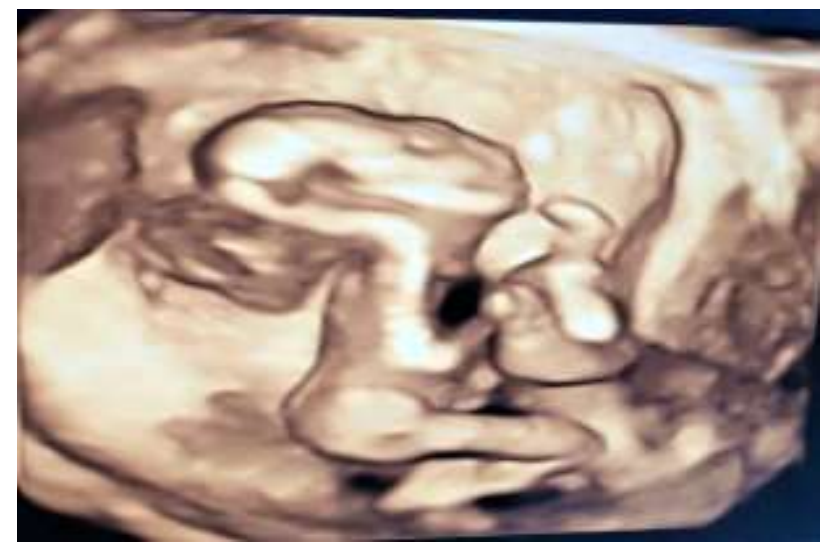

Figure 2: Encephalocele

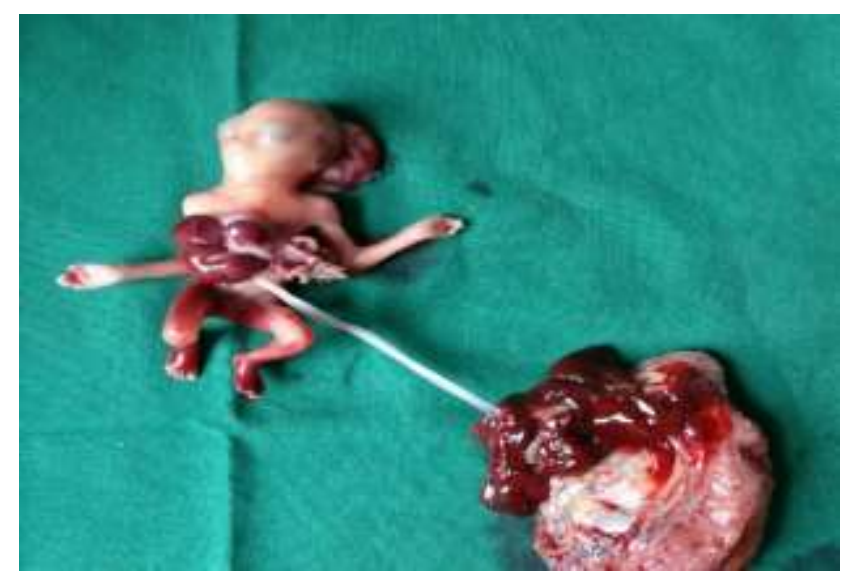

Figure 3: Expelled fetus with anomalies

\section{DISCUSSION}

Body stalk anomaly is a rare condition with an incidence rate of 1 in 7500 foetuses at 10 to 14 weeks of gestation according to a multicentric study conducted in London and primarily is due improper embryonic folding. ${ }^{3}$ Body stalk anomaly is characterised by anterior body wall defect associated with extrusion of thoracic and abdominal organs, kyphoscoliosis, absent or short umbilical cord, limb deformities. Maternal serum alpha protein may be increased. Normally during $5^{\text {th }}$ week of gestation, the developing placenta is connected to the trilaminar embryo by means of a body stalk. The embryo is transformed into a cylindrical form by folding in all three axes namely caudal, lateral and cephalic. Body stalk anomaly is caused due to improper folding in all the three axes associated with abnormality in the amniotic sac and failure of coelomic cavity obliteration. Various other hypothesis was stated to explain the incidence of body stalk anomaly. Evidence of early amnion rupture prior to coelomic cavity obliteration. The abdominal wall and spine defects were due to passage of lower half of the fetal body through the defect in the amniotic sac into the coelomic cavity. Encephalocele and amputation of limb can occur as a result of entrapment of the skull and fetal limbs in the coelomic cavity respectively. Growth of the umbilical cord occurs due to tensile forces and depends on the fluid spaces and fetal movements. Any condition that reduces the space or the restricts the fetal movements may result in reduction in the length of the cord. Due to compromised blood flow to the embryo in early pregnancy, closure of ventral body wall fails to occur and the coelomic cavity persists. This may cause rupture of the amniotic cavity. ${ }^{4,2}$

Diagnosis of body stalk anomaly is primarily by 2dimensional or 3-dimensional sonography performed at early second which helps in ascertaining the precise relationship of the fetal structures to the amniotic cavity. According to Murphy et al, 4 cases of body stalk anomaly were identified. 3-dimensional sonography was more accurate in arriving at the diagnosis of body stalk anomaly. Using multiplanar assessment and parallel shifting techniques exact anatomical location, their relation to the amniotic cavity and for identifying other associated anomalies. ${ }^{5}$ A nationwide epidemiological study of 20 annual Danish birth cohorts from 1970 to 1989 and was compiled in a registry which took into account 469 live and still born infants. This study identified 16 cases of body stalk deformity. According to Martinez-Frias et al, the parental age of foetuses with body stalk deformity is lower than in the control group. The sex ratio was 1.5 but in majority cases the sex was indeterminate. Gestational age and birth weight was reduced when compared to the control group. ${ }^{6}$ According to Hunter et al, all the infants were tested for karyotype and was found to be normal. ${ }^{7}$

\section{CONCLUSION}

Body stalk malformation is a lethal condition either in utero or in early neonatal period which can be diagnosed in early trimester ultrasound. The association of chromosomal abnormalities and genetic syndromes in 
this condition is rare and the risk of recurrence in next pregnancy is less.

Funding: No funding sources Conflict of interest: None declared

Ethical approval: Not required

\section{REFERENCES}

1. Panaitescu AM, Ushakov F, Kalaskar A, Pandya PP. Ultrasound Features and Management of Body Stalk Anomaly. Fetal Diagn Ther. 2016;40(4):285-90.

2. Smrcek JM, Germer U, Krokowski M, Berg C, Krapp M, Geipel A et al. Prenatal ultrasound diagnosis and management of body stalk anomaly: analysis of nine singleton and two multiple pregnancies. Ultrasound Obstet Gynecol. 2003;21(4):322-8.

3. Daskalakis G, Sebire NJ, Jurkovic D, Snijders RJM, Nicolaides KH. Body stalk anomaly at 10-14 weeks of gestation. Ultrasound Obstet Gynecol. 1997;10(6):416-8.
4. Bugge M. Body stalk anomaly in Denmark during 20 years (1970-1989). Am J Med Genet A. 2012;158A(7):1702-8.

5. Murphy A, Platt LD. First-trimester diagnosis of body stalk anomaly using 2- and 3-dimensional sonography. J Ultrasound Med Off $\mathbf{J}$ Am Inst Ultrasound Med. 2011;30(12):1739-43.

6. Martínez-Frías ML, Bermejo E, Rodríguez-Pinilla E. Body stalk defects, body wall defects, amniotic bands with and without body wall defects, and gastroschisis: comparative epidemiology. Am J Med Genet. 2000;92(1):13-8.

7. Stevenson RE, Rogers RC, Chandler JC, Gauderer MWL, Hunter AGW. Escape of the yolk sac: a hypothesis to explain the embryogenesis of gastroschisis. Clin Genet. 2009;75(4):326-33.

Cite this article as: Balachandran R, Samal SK.

Fetal body stalk malformation-a rare case report. Int $\mathrm{J}$ Reprod Contracept Obstet Gynecol 2021;10:394-6. 